\title{
PRIME RINGS SATISFYING A POLYNOMIAL IDENTITY
}

\author{
EDWARD C. POSNER
}

THEOREM. $R$ is a prime ring satisfying a polynomial identity if and only if $R$ is a subring of the ring of all $r \times r$ matrices, for some $r$, over a division ring $D$ finite dimensional over its center, and $R$ has a two-sided ring of quotients which is all of the matrix ring. (For this result when $R$ has no zero divisors, see [1]).

Proof. Sufficiency is easy and omitted. We recall that $B$ is a twosided quotient ring of its subring $A$ if every element of $B$ can be written $a b^{-1}, a, b \in A$, and also $c^{-1} d, c, d \in A$, and if every element of $B$ not a left (right) zero divisor has a right (left) inverse in $B . B$ is unique given $A$ if it exists.

To prove necessity we invoke Goldie's Theorem (2): Let $R$ be a prime ring satisfying (1l), (1r), (2l), (2r). Then $R$ has an $m \times m$ matrix ring over a division ring as its full ring of quotients. Here $(1 l)$ is: every direct sum of nonzero left ideals of $R$ has a finite number of terms. (2l) is: the ascending chain condition holds for the annihilator left ideals of $R$. (1r) and (2r) are analogous.

We will prove that if $R$ is a prime ring satisfying a polynomial identity, then $R$ satisfies ( $1 l)$ and $(2 l)$. The conditions for right ideals will follow similarly. Let $\sum a_{\pi} x_{\pi(1)} \cdots x_{\pi(n)}=0, a_{\pi} \in C$, the centroid of $R, \pi$ a permutation of $1,2, \cdots, n$, be a homogeneous multilinear identity for $R$. We will show that the length of a direct sum of nonzero left ideals is at most $n-1$. First let $I_{j}, 1 \leqq j \leqq n$, be left ideals invariant under the centroid of $R$, and let $I_{1} \oplus \cdots \oplus I_{n}$ be direct. Let $x_{j} \in I_{j}$. Then all terms in the identity whose rightmost factor is $x_{1}$, say, must add up to zero by directness and the fact that the $I_{j}$ are invariant under the centroid. Here $x_{1}$ was so numbered that at least one nonzero coefficient occurs. Since $R$ is prime, $I_{1}$ has no left annihilator, that is, we can now cancel $x_{1}$ from this identity. Continuing in this fashion, renumbering if necessary, we find $I_{n}=0$. If $I_{j}$ are not invariant under the centroid $C$, the $C I_{j}$ are, and $C I_{1} \oplus \cdots \oplus C I_{n}$ is still direct. For if $\sum_{j=1}^{n} c_{j} i_{j}=0, c_{j} \in C, i_{j} \in I_{j}, 1 \leqq j \leqq n$, then $\forall r \in R$, $\sum_{j=1}^{n}\left(r c_{j}\right) i_{j}=0$. But $r c_{j} \cdot i_{j} \in I_{j}, 1 \leqq j \leqq n$, so each $r c_{j} i_{j}=0$. Since $R$ has no absolute right divisors of zero, $c_{j} i_{j}=0,1 \leqq j \leqq n$.

To prove that $R$ satisfies the ascending chain condition for left annihilator ideals, suppose $I_{1} \Phi I_{2} \Phi \cdots \Phi I_{n}$ are left annihilator

Received by the editors April 25, 1959. 
ideals where we may suppose $I_{j}$ is the total left annihilator of a right ideal $K_{j}$ and $K_{j} \neq K_{j-1}, 2 \leqq j \leqq n$. Let $k(\leqq n)$ be the first integer such that $\exists\left\{\beta_{\pi}\right\} \in C$ not all zero with $\sum \beta_{\pi} i_{\pi(1)} \cdots i_{\pi(k)}=0$ whenever $i_{j} \in I_{j}, 1 \leqq j \leqq k$, the sum extended over all permutations of $1,2, \cdots, k$, and such that $\beta_{\pi} \neq 0$ when $\pi$ is the identity permutation. Multiply this identity on the right by $K_{k-1} . \sum^{\prime} \beta_{\pi} i_{\pi(1)} \cdots i_{\pi(k-1)} i_{\pi(k)} K_{k-1}=0$ where $\Sigma^{\prime}$ is taken over those $\pi$ with $\pi(k)=k$. In other words $\sum \beta_{\pi} i_{\pi(1)} \cdots i_{\pi(k-1)} i_{k} K_{k-1}=0$, the sum extended over all permutations of $1,2, \cdots, k-1, \forall i_{k} \in I_{k}$. Or $\sum \beta_{\pi} i_{\pi(1)} \cdots i_{\pi(k-1)} I_{k} K_{k-1}=0$, $\forall i_{j} \in I_{j}, 1 \leqq j \leqq k-1$. Now $I_{k} K_{k-1}$ is a two-sided ideal, and, by assumption, not zero. So by primeness, $\sum \beta_{\pi} i_{\pi(1)} \cdots i_{\pi(k-1)}=0$, which contradicts the minimality of $k$. So the assumption that $I_{k} I_{k-1} \neq 0$ must be retracted, $I_{k-1}=I_{k}$ and not $I_{k-1} \Phi I_{k}$.

To prove that the quotient ring $Q$ of $R$ is a matrix ring over a finite-dimensional division ring, it suffices to prove that $Q$ satisfies a polynomial identity. (Actually $Q$ satisfies the same identity as we shall see.) $R$ satisfies a standard identity $\sum \operatorname{sgn} \pi x_{\pi(1)} \cdots x_{\pi(2 p)}=0$ where sgn $\pi$ is +1 or -1 according as $\pi$ is even or odd; in fact $R$, having no nilpotent ideals, is a subring of a direct sum of $p \times p$ matrix rings over fields $[3$, p. 227 , Theorem 2]. We wish to prove $\sum \operatorname{sgn} \pi x_{\pi(1)} d_{\pi(1)}^{-1} \cdots x_{\pi(2 p)} d_{\pi(2 p)}^{-1}=0$ for all $x_{i}, d_{i} \in R, d_{i}$ regular in $R$, $1 \leqq i \leqq 2 p$. But resorting to the very definition of rings of quotients $[4$, p. 118], we can write the condition that the standard identity of degree $2 p$ be satisfied in a form not involving inverses at all by resorting to the definition of addition and multiplication in $Q$. The condition is of the form that if a certain (large) set of auxiliary elements of $R$ satisfy one set of equations involving the $x_{i}$ and $d_{i}$, and not their inverses, (namely, the set of equations which describes when $u v^{-1}, u, v \in R$ involving the $x_{i}, d_{i}$ and auxiliary elements, is $\sum \operatorname{sgn} \pi x_{\pi(1)} d_{\pi(1)}^{-1} \cdots x_{\pi(2 p)} d_{\pi(2 p)}^{-1}$ in $\left.Q\right)$, then they satisfy another equation, namely, $u=0$. For $p \times p$ matrices over fields, we know that whenever the first set of equations is satisfied by auxiliary elements, the second equation is satisfied, since $p \times p$ matrices satisfy the standard identity of degree $2 p$. That is, the second equation is satisfied if the $d_{i}$ were invertible in the matrix ring. (Our $d_{i}$ in $R$ may not be invertible in each matrix summand in which $R$ is embedded, where invertible means invertible as a matrix and not as an element of $R$.) We wish to show that the second equation is satisfied even if the $d_{i}$ are not invertible matrices. Let the coefficients in the $2 p p \times p$ matrices $d_{i}$ be $2 p$ independent transcendentals $\left\{y_{\gamma}\right\}$. In particular, each $d_{i}$ is invertible. We may keep the $x_{i}$ fixed at their given values. To say that $u=0$ is satisfied whenever the other set of equations is 
satisfied says that the set of zeros of the $p^{2}$ polynomials $\left\{f_{\alpha}\right\}$ obtained from $u=0$ in the indeterminates $\left\{u_{\delta}\right\}$ (corresponding to the coefficients of the matrices of each auxiliary variable) contains the set of zeros of the other set of polynomials $\left\{g_{\beta}\right\}$ (obtained from the set of equations defining $u$ ). By Hilbert's Nullstellensatz, for some integer $q$ which we may take to be the same for every $\alpha, f_{\alpha}^{q}=\sum r_{\beta, \alpha} g_{\beta}$, all $\alpha$, where the $r_{\beta, \alpha}$ are also polynomials in the $\left\{u_{\delta}\right\}$. Now these polynomials involve the $\left\{y_{\gamma}\right\}$ as parameters. Let $\left\{\bar{f}_{\alpha}\right\},\left\{\bar{r}_{\beta, \alpha}\right\},\left\{\bar{g}_{\beta}\right\}$ be the corresponding polynomials when the $\left\{y_{\gamma}\right\}$ are specialized to $\left\{w_{\gamma}\right\}$ say, where the $\left\{w_{\gamma}\right\}$ arise from a set $\left\{d_{i}\right\}$ of not necessarily invertible matrices. Then $\bar{f}_{\alpha}^{q}=\sum \bar{r}_{\beta, \alpha} \bar{g}_{\beta}$. But this means that all $\bar{f}_{\alpha}$ are zero if all $\bar{g}_{\beta}$ are zero. Referring to the meeting of $\left\{\bar{f}_{\alpha}\right\},\left\{\bar{g}_{\beta}\right\}$, we conclude that whenever the first set of equations is satisfied by elements of a $p \times p$ matrix algebra, then so is the second, as promised. $R$ is a subring of a direct sum of $p \times p$ matrix rings over fields, so the conclusion of the preceding sentence holds for $R$ also. And in case the $\left\{d_{i}\right\}$ are now (ring) invertible elements of $R$, we can reverse the process and conclude that $Q$ satisfies the standard identity of degree $2 p$. Thus the theorem is proved.

To prove that $Q$ satisfies the original multilinear homogeneous equation that $R$ satisfied, consider $R_{1}$, which is $R$ written with coefficients from the center $F$ of $Q . R_{1}$ is prime, since $R_{1}$ has $Q$ as a twosided ring of quotients. $R_{1}$ is finite dimensional over $F$ since $Q$ is. Then $R_{1}$ is a finite dimensional simple algebra and hence is its own ring of quotients. So $R_{1}=Q$. But $R_{1}$ satisfies the original identity and therefore $Q$ does.

Corollary. Let $A$ be an algebra satisfying a polynomial identity over its field and such that every element of $A$ is a sum of nilpotent elements. Then $A$ is nil.

Proof. We show $A$ has no prime quotients, thus proving $A$ is its own lower nil radical. We remark that a prime ideal, in fact any ideal modulo which there are no nilpotent ideals, is an algebra ideal, so that a prime quotient $R$ of $A$ is also a polynomial identity algebra in which every element is a sum of nilpotents. But by the last part of the theorem, $\left(R_{1}=Q\right)$, the quotient matrix algebra $Q$ of $A$ also has this property, and the following known argument completes the proof. $Q$ is a total matrix algebra over a division algebra $D$ finite dimensional over its center $F$. Let $K$ be a splitting field for $D$ over $F$ so that $Q_{1}=Q \otimes_{F} K$ is a total matrix algebra over $K$. Note that in $Q_{1}$, every element is still a sum of nilpotent elements, so that every element of $Q_{1}$ has trace zero. And yet the matrix with a 1 in the $(1,1)$ 
position and zeros elsewhere does not have trace zero. Thus the corollary is proven.

We remark that the corollary is true for rings with identities with one term having \pm 1 as coefficient, but is false for arbitrary polynomial identity rings. For Harris [5] has produced a total $2 \times 2$ matrix ring over a division ring which we may take to be of characteristic 2 in which every element is a sum of nilpotent elements. The direct sum of this with a trivial algebra of characteristic 0 satisfies $2 x_{1} x_{2}=0$ but is not nil.

\section{BIBLIOGRAPHY}

1. S. Amitsur, On rings with identities, J. London Math. Soc. vol. 30 (1955) pp. 464-470.

2. A. W. Goldie, The structure of prime rings under ascending chain conditions, Proc. London Math. Soc. vol. 8 (1958) pp. 589-608.

3. N. Jacobson, Structure of rings, Amer. Math. Soc. Colloquium Publications, vol. $37,1956$.

4. - The theory of rings, Mathematical Surveys, 1943.

5. B. Harris, Commutators in divison rings, Proc. Amer. Math. Soc. vol. 9 (1958) pp. 628-630.

UNIVERSITY OF Wisconsin, MADISON 